\title{
Un réseau de voies d'accès à la langue spécialisée en anglais L2
}

Catherine Resche

\section{(2) OpenEdition}

Édition électronique

URL : http://journals.openedition.org/asp/2601

DOI : 10.4000/asp.2601

ISBN : 978-2-8218-0380-0

ISSN : 2108-6354

Éditeur

Groupe d'étude et de recherche en anglais de spécialité

Édition imprimée

Date de publication : 1 décembre 1999

Pagination : 349-373

ISSN : 1246-8185

Référence électronique

Catherine Resche, «Un réseau de voies d'accès à la langue spécialisée en anglais L2 », ASp [En ligne], 23-26 | 1999, mis en ligne le 04 janvier 2012, consulté le 19 avril 2019. URL : http://

journals.openedition.org/asp/2601; DOI : 10.4000/asp.2601

Ce document a été généré automatiquement le 19 avril 2019

Tous droits réservés 


\title{
Un réseau de voies d'accès à la langue spécialisée en anglais L2
}

\author{
Catherine Resche
}

1 Nous voudrions tout d'abord insister sur le choix du terme "langue spécialisée », de préférence à « langue de spécialité » en faisant référence au titre du livre de Pierre Lerat (1995), qui illustre le fait que la langue sur laquelle nous travaillons avec nos étudiants dans le secteur LANSAD n'est pas un sous-système, une langue à part, mais bien en premier lieu de l'anglais. L'anglais économique, pour prendre un exemple qui nous est plus familier, plonge bien ses racines dans l'anglais général, même s'il est plus particulièrement le «vecteur de savoirs et de savoir-faire» (Lerat 1995: 11-12). Ce message est également celui qui ressort de la pyramide de la langue spécialisée établie par Michel Perrin (1990: 27) et adaptée à la langue économique par nos soins en y ajoutant une strate supplémentaire (Resche 1995: 94) sur laquelle nous reviendrons dans la dernière partie de cette étude. Chaque composante du terme «langue spécialisée » est donc également importante : nous avons le double rôle, d'une part, de nous assurer que nos étudiants ont une bonne maitrise de l'anglais et, d'autre part, de faire en sorte de leur donner les moyens d'étudier et d'approfondir la langue d'un domaine bien particulier, avec tout ce que cela signifie en terme de technicité et d'authenticité, afin que, dans un cas de figure idéal, ils aient accès à la communauté qui utilise cette langue et soient reconnus par elle. De manière plus réaliste, nous devons tendre vers ce but, en leur faisant acquérir les méthodes qui leur permettront de se familiariser avec cette langue qui ne doit plus constituer un obstacle dans leurs futurs travaux de recherche ou dans leur carrière à venir.

2 Dans les années passées, nous avons eu l'occasion d'exposer diverses approches de l'anglais de l'économie et de la finance, et il est temps maintenant de faire le point sur ces voies d'accès plurielles, complémentaires et interconnectées, qui offrent autant d'angles pour aborder la langue spécialisée. Nous proposons donc d'examiner en premier lieu, la voie de la lecture, parce qu'elle représente le premier accès aux textes en LSP, parmi lesquels les ouvrages de référence, souvent rédigés en anglais, et qui sont un passage 
obligé pour les étudiants (en 2e et 3e cycles, par exemple). Lire plus facilement permet, en effet, de lire pour apprendre, pour mettre à jour des connaissances, pour chercher des informations, pour vérifier des faits, etc. Nous examinerons, au passage, les techniques qui peuvent être mises en œuvre pour aider à la compréhension des textes, pour en appréhender l'organisation, ce qui nous conduira à illustrer un certain nombre de réseaux internes.

Ensuite, nous reviendrons sur ce qui constitue " par excellence le matériau distinctif du texte spécialisé » (Lerat 1995 : 62), à savoir la terminologie propre au domaine étudié et son complément, la phraséologie, qui, toutes deux, entretiennent avec la lecture des liens privilégiés : d'une part, termes et phrasèmes se repèrent en contexte, donc grâce à la lecture ; d'autre part, la connaissance de ces groupes de plus en plus longs permet de lire par groupes de sens, et logiquement de lire plus vite, en comprenant mieux le sens de la chose écrite.

4 Toutefois, il ne faut pas négliger les aspects syntaxiques, ni les différents types de textes auxquels les étudiants seront confrontés. Nous aborderons donc ces problèmes, tout en leur réservant une étude plus complète pour une analyse ultérieure. Enfin, nous nous intéresserons à des approches peut-être plus caractéristiques de la langue spécialisée liée aux sciences humaines, en nous attachant plus particulièrement aux bénéfices que l'on peut tirer de l'observation des néologismes, des métaphores et des dictons rencontrés, sans oublier les références plus ou moins directes à divers genres littéraires, qui permettent d'appréhender la langue spécialisée dans sa dimension culturelle.

Pour des besoins de clarté, la présentation des différentes approches requiert une étude séparée mais il ne faudrait pas pour autant y voir un cloisonnement entre les genres, car, bien au contraire, toutes les approches se chevauchent et s'enrichissent mutuellement, comme le schéma suivant permet de l'illustrer :

Fig.1. Réseau des chemins d'accès à la langue spécialisée

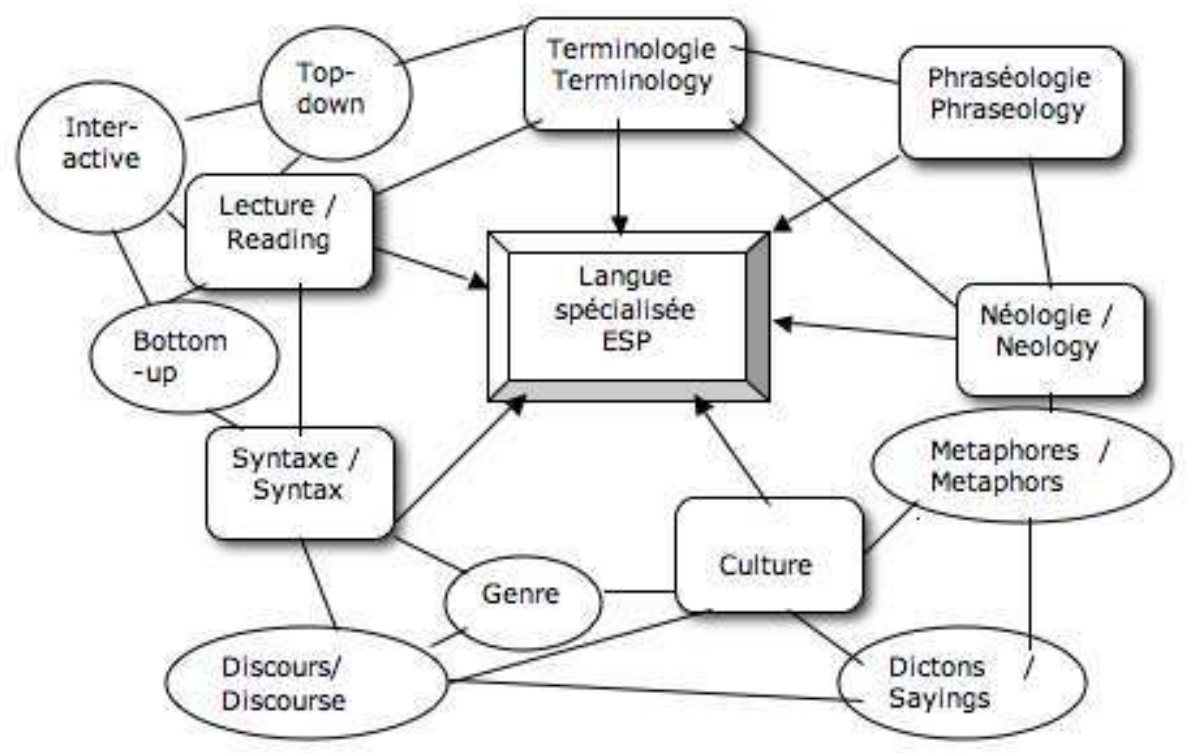

6 Il est cependant logique de commencer par la lecture compréhension car la langue spécialisée s'illustre dans des textes et contextes. 


\section{L'approche de la lecture en langue spécialisée}

7 Un texte est en lui-même un ensemble de mots, de syntagmes formant des phrases simples ou composées, reliées entre elles pour constituer des paragraphes, eux-mêmes structurés les uns par rapport aux autres. Il ressort de ces différents niveaux de construction qui s'emboîtent les uns dans les autres qu'il peut y avoir plusieurs points d'accès, que nous nous proposons de rappeler tour à tour.

\subsection{L'approche « bottom-up »}

8 On peut en effet s'attacher au plus petit élément mentionné ci-dessus, à savoir le mot, pour passer progressivement aux échelons supérieurs et arriver ainsi à extraire le sens à partir des données du texte. Cette démarche, résumée par Patricia Carrell (1992:2) correspond à un décodage de la chose écrite :

a decoding process of reconstructing the author's intended meaning via recognizing the printed letters and words, and building up a meaning for a text from the smallest textual units at the "bottom" (letters and words) to larger units at the "top" (phrases, clauses, intersentential linkages).

9 Bon nombre d'exercices pratiqués par une majorité d'enseignants reflètent plus ou moins sciemment certaines de ces préoccupations. Tout ce qui vise la reconnaissance de mots dans une série, ou de manière un peu plus sophistiquée, l'identification de rapports sémantiques, de synonymie ou d'antonymie entre les mots, relève de ces principes. La recherche de mots clés dans une phrase, le repérage du noyau essentiel sujet/verbe, la dissection de termes composés, l'extraction de racines connues font également partie de cette entreprise de décodage. Selon le niveau du public auquel on a affaire, certaines des étapes pourront être survolées mais, pour les plus faibles, des exercices préparatoires au niveau du lexique s'avèrent souhaitables. Toutefois, plutôt que de travailler à partir de listes de vocabulaire rébarbatives, il sera préférable de s'attacher à montrer dès le départ que les mots ne sont pas à considérer isolément. En effet, ils se définissent in praesentia selon l'axe syntagmatique, c'est-à-dire par rapport aux autres composantes de la phrase, aussi bien que in absentia, par substitution possible avec d'autres termes selon l'axe paradigmatique. On peut d'ailleurs envisager, pour matérialiser ces associations diverses et travailler sur des champs sémantiques plus larges, d'établir des schémas à partir d'un terme central autour duquel va se tisser la toile de la combinatoire sémantique par simple association d'idées (synonymes, antonymes, domaines d'application, etc.).

Pour donner une idée du travail qui peut être fait dans ce sens, nous nous sommes livrée à ce petit jeu en annexe 1, à partir du terme « réseau » en français, ce qui nous a permis de nous rendre compte que le champ couvert correspondait en anglais à la fois aux mots centres «network» et " net », auxquels sont rattachés "grid» et «lattice». Ce type de travail est fructueux en ce qu'il permet d'élargir les horizons lexicaux et d'envisager maintes interprétations auxquelles les mots clés peuvent donner lieu, selon les domaines d'application. Cela peut servir de point de départ à une étude de la polysémie, qui, faut-il le souligner, existe même à l'intérieur de la langue spécialisée, dont il convient alors d'explorer les sous-domaines.

11 Si les étudiants ont un bagage lexical suffisant, c'est peut-être au niveau des syntagmes qu'il faut travailler, niveau plus intéressant surtout en langue de spécialité, et nous y 
reviendrons en traitant des approches terminologique et phraséologique. Quant au niveau supérieur, celui de la phrase simple ou composée, il met en relief deux points importants pour le travail sur la lecture qui touchent à la forme et au fond. D'une part, il souligne que la phrase n'est pas simplement une juxtaposition de mots mais que les mots y sont ordonnés en fonction de structures lexicales ou grammaticales dont il faut avoir la maîtrise. D'autre part, pour accéder au sens de la phrase, il faut également en comprendre l'organisation interne qui préside à l'enchaînement logique des faits énoncés et des propositions. Dans le même ordre d'idées, il ne suffit pas que les phrases se suivent et que les paragraphes soient juxtaposés pour constituer un texte. Les maîtres mots étant cohésion et cohérence, il faut aider les étudiants à être sensibles aux indices et à retrouver la trame du texte s'ils veulent arriver à la compréhension de l'ensemble. Il faudra donc que l'enseignant choisisse des textes abordables par les étudiants sur le plan scientifique, afin que la part faite à l'implicite n'y soit pas trop grande, ce qui les dérouterait par une impression d'incohérence.

S'agissant de la cohésion, là encore, les exercices préparatoires sont largement pratiqués par les uns et les autres (Fade 1994: 122), qu'il s'agisse de familiariser les étudiants avec les éléments anaphoriques ou cataphoriques, pronoms, déterminants démonstratifs, possessifs, indéfinis, numéraux, marqueurs de temps, de relations (cause/effet, etc.), synonymes et antonymes présents dans le contexte. Dans la progression vers les unités de plus en plus larges que suggère l'approche "bottom-up ", les étudiants sont ensuite amenés à chercher dans le texte les phrases clés, les idées phares, par exemple en se fondant sur la première phrase de chaque paragraphe et à analyser la hiérarchisation des paragraphes. Ceci aide à les sensibiliser à la structure des textes et à leur faire apprécier différents genres (Swales 1990: 89) et ce travail peut se faire en leur demandant de schématiser cette structure sous forme de diagramme (voir annexe 2).

Generally speaking, text mapping involves selecting key content from an expository passage and representing it in some sort of visual display (boxes, circles, connecting lines, tree diagrams, etc.) in which the relationships among the key ideas are made explicit. (Carrell $1992: 249$ )

\subsection{L'approche «top-down »}

13 Le reproche qui a été fait à l'approche précédemment décrite est qu'elle entraîne une trop grande passivité de la part du lecteur qui tire toute l'information du texte et qu'elle risque de lasser ou décourager quiconque a des difficultés à accéder aux échelons supérieurs. On concevra, en effet, que si l'on bute sur les mots, le travail sera vite fastidieux et l'analyse détaillée, au même titre que le découpage au mot accompagné d'un recours systématique au dictionnaire, entraveront la vue d'ensemble.

La réponse à ce problème a été avancée par les tenants de l'approche « top-down » qui se fondent sur le principe que lire revient à émettre des hypothèses à partir d'indices, et à les vérifier en avançant dans le texte, ce qui permet de confirmer ou d'infirmer ces hypothèses :

In the top-down view of second-language reading, not only is the reader an active participant in the reading process, making predictions and processing information, but everything in the reader's prior experience or background knowledge plays a significant role in the process. In this view, not only is the reader's prior linguistic knowledge ('linguistic schemata') and level of proficiency in the second language important, but the reader's prior background knowledge of the content area of the 
text ('content schemata') as well as of the rhetorical structure of the text ('formal

schemata') are also important. (Carrell $1992: 4)$ vérifie mieux dans la mesure où ils peuvent davantage compter sur leur connaissance pointue du domaine étudié pour pallier des lacunes sur le plan lexical ou structurel. Ils sont en effet en possession de schémas logiques d'associations d'idées qui doivent leur servir de repères quand ils abordent un sujet particulier. Une fois qu'ils savent quel est le thème traité, qu'ils ont réfléchi aux termes clés, ils vont pouvoir s'appuyer sur leurs connaissances préalablement structurées pour construire des hypothèses, les vérifier, en rejeter certaines, en émettre d'autres, etc. :

The reader starts with the perception of graphic cues, but as soon as these are recognized as familiar, schemata derived from both linguistic knowledge and knowledge of the world in general are brought into play. (Parry, 1987, cité par Eskey \& Grabe, 1992 : 225)

C'est ainsi que la cooccurrence de deux termes, par exemple, «global financial turmoil / slowdown » va suffire à réactiver un schéma déjà intégré, comme le montre l'exemple suivant (Fig.2) :

Fig. 2. Schéma mental probable à partir des notions " ralentissement de l'économie » et « tempête financière à l'échelle planétaire »

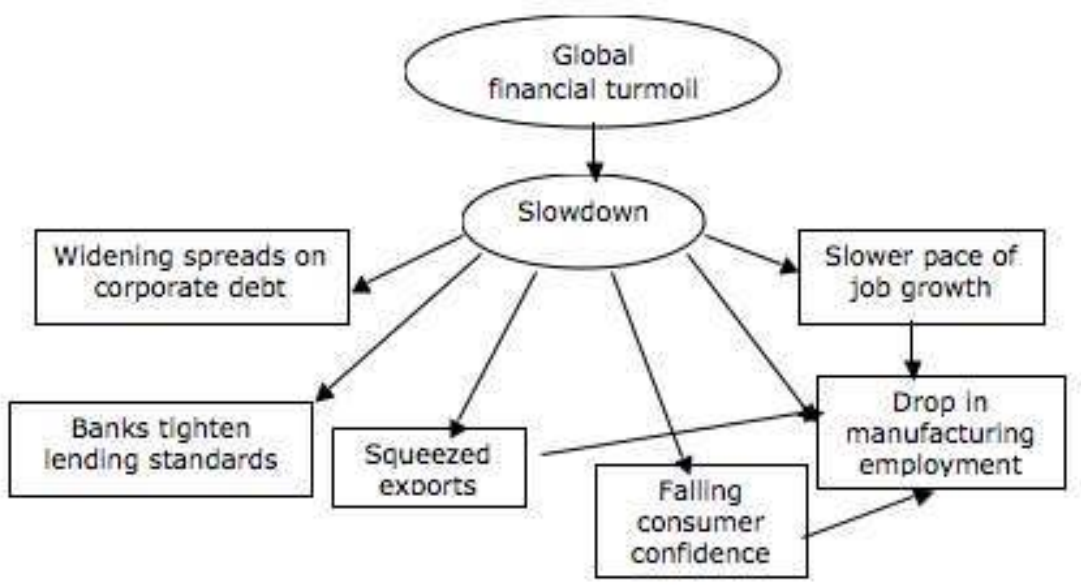

ASp, 23-26 | 2011 
19 Au fur et à mesure qu'il lira le texte, l'étudiant va confronter les informations avec le schéma mental présenté ci-dessus, s'attendant à rencontrer tel ou tel autre point, ce qui va l'aider à privilégier certains développements, déduire le sens de certains termes ou groupes de mots moins clairs, et éviter des contresens.

\subsection{L'approche interactive}

Efficient and effective reading -it in a first or second language-requires both topdown and bottom-up strategies operating interactively. (Carrell $1992: 4)$.

C'est au sens de complémentaire et d'alternatif qu'il faut comprendre « interactif », c'està-dire que les deux approches décrites précédemment ne doivent par être mutuellement exclusives mais doivent être envisagées comme recours à tout moment susceptible de fonctionner là ou l'autre ne répond pas aux besoins ponctuels. De cette manière, on peut envisager qu'un étudiant de première année en économie devra davantage compter sur les données du texte car il a encore peu de connaissances spécialisées sur les sujets abordés, alors qu'un étudiant de troisième cycle aura une plus grande maîtrise des questions et mécanismes évoqués. Cependant, indépendamment de cela, le niveau en langue des étudiants ne va pas toujours de pair avec leur progression dans leur spécialisation, de sorte qu'il faut leur donner les moyens de recourir à l'une ou l'autre de ces approches en fonction des difficultés qu'ils rencontrent.

Interactive models...assume that a pattern is synthesized based on information provided simultaneously from several knowledge sources. The compensatory assumption states that a deficit in any knowledge results in a heavier reliance on other knowledge sources, regardless of their level in the processing hierarchy. (Stanovitch $1990: 63$ )

21 Le schéma à la figure 3 permettra de mettre en relief la complémentarité des deux approches.

Fig.3. Approches complémentaires pour la compréhension d'un texte

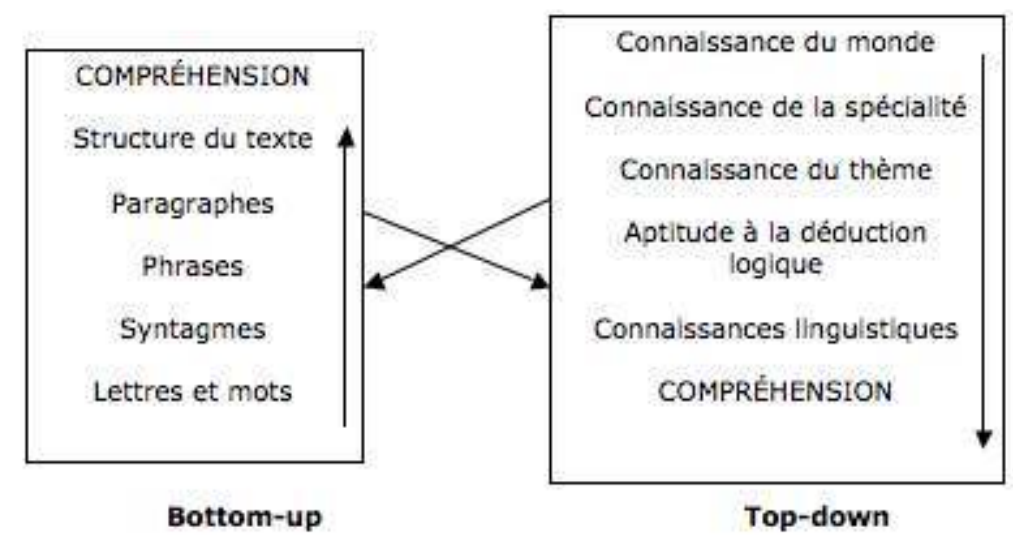

Quelle que soit l'approche mise en œuvre, l'important est de faire en sorte que le lecteur lise de plus en plus efficacement et dans diverses optiques, comme dans sa propre langue, c'est-à-dire soit pour apprendre, pour trouver des informations précises, soit pour survoler le texte afin de déterminer s'il correspond à ce qu'il cherche, soit encore pour pouvoir confronter plusieurs points de vue et jeter un regard critique sur les arguments avancés : 
Students must learn to skim for the main idea and to scan for specific kinds of information and they may also have to learn to read critically, to make informed evaluations of an author's arguments. (Eskey \& Grabe 1992 : 229) une communauté donnée qui partage un savoir et un savoir-faire et qui, pour des besoins de communication évidents, a recours à des termes établis pour dénommer selon les cas et les spécialisations, des outils, des mécanismes ou des concepts. Comme cette terminologie a pour but d'éviter les problèmes «d'ésotérisme, de babélisation et de lacunes lexicales » (Lerat 1995 : 108), elle est caractérisée par un besoin de définition, de classification, de nomenclature (Guilbert 1973 : 10-11). Il importe donc de faire en sorte que les étudiants qui se spécialisent dans un secteur particulier puissent s'approprier la terminologie en usage chez les praticiens et techniciens du domaine, ce qui sous-entend qu'ils doivent avoir la maîtrise des concepts sous-jacents, aussi bien dans leur langue maternelle qu'en anglais, langue étrangère. Le cas échéant, ils sont également censés être conscients des différences, de la correspondance imparfaite de certaines notions. terminologiques en les habituant à se fonder sur les surfaces conceptuelles des notions. Le lien avec la méthodologie exposée plus haut quant à la lecture est évident dans la mesure où l'on veut leur apprendre à lire par groupes de sens. Apprendre à repérer les termes, qui, nous le rappelons, sont la dénomination de concepts revient donc à faire un premier pas vers ces groupes de sens puisque le terme est bien souvent un composé syntagmatique, une unité de signification caractérisée par « la relation biunivoque entre un mot ou un groupe de mots et une définition spécialisée » (Lerat 1995 : 49). Nous ne reviendrons pas sur la nécessité de bien différencier les déterminants relationnels des déterminants accidentels (Resche 1995 : 102), ni sur les moyens à mettre en œuvre pour éviter les pièges et les approximations.

Une fois qu'ils sont en possession des termes dont ils ont vérifié le bien-fondé en s'assurant de leurs définitions, les étudiants doivent savoir quelle place ces termes occupent les uns par rapport aux autres afin de structurer ces nouvelles données et de les intégrer aux connaissances déjà acquises. Il devient alors possible d'établir des arborescences par champs notionnels, qui correspondent à la hiérarchisation des concepts. Ces arborescences, qui matérialisent la mise en réseau des termes d'un domaine, permettent de vérifier la logique des liens internes et de clarifier les notions, aidant ainsi à l'appropriation des termes.

Comme nous avons eu l'occasion de l'expliquer en d'autres circonstances (Resche 1996), il est tout à fait possible d'initier des étudiants de troisième cycle à l'élaboration d'un dossier terminologique sur un domaine bien délimité, ce qui les amène à lire encore davantage pour trouver les différents renseignements qui constitueront la fiche 
terminologique, véritable carte d'identité du terme vedette: définition, contexte pertinent, notes concernant les synonymes et antonymes éventuels, les variantes orthographiques, géographiques, les renvois à d'autres termes, le niveau de technicité, etc. En fait, «mérite d'être pris en considération tout ce qui n'est pas prédictible à partir soit d'une compétence linguistique générale, soit des connaissances de sens commun » (Lerat 1997 : 7).

En revanche, exiger ce type de travail en premier cycle n'est pas possible, car le degré de spécialisation est moindre et les conditions de travail, ne serait-ce que la taille des groupes, ne s'y prêtent guère. Toutefois, il n'est pas inutile de commencer à insister sur la notion d'unité terminologique, de demander aux étudiants quels sont les groupes de mots qui constituent des termes à leurs yeux dans un texte donné, et de discuter le bien-fondé de leurs choix. Ceci les conduit vite à prendre conscience de l'incongruité qu'il y aurait à essayer de traduire d'une langue dans l'autre sans se soucier de l'authenticité de la langue et cela les oblige à s'imposer une certaine rigueur qui ne peut être que bénéfique dans l'expression écrite comme orale.

Le travail en terminologie a également pour effet de mieux faire comprendre aux étudiants pourquoi les spécialistes, paradoxalement, n'éprouvent pas le besoin de spécifier à outrance les notions lorsqu'ils s'adressent à leurs pairs, alors que leur discours se fera plus précis et contiendra davantage de contextes définitoires dès lors qu'ils s'expliquent devant un public plus hétérogène. Ils font eux-mêmes l'expérience de termes communs à la langue générale et à la langue spécialisée qui ne prêtent pas à confusion, en dépit de leur polysémie, si l'on connaît le domaine restreint dans lequel ils sont utilisés : par exemple, instrument ou tool seront immédiatement compris à leur juste sens dans un contexte économique et, dans ce cas, toute ambiguïté disparaitra. C'est souvent la cooccurrence de deux termes qui suffira à clarifier le sens pour l'initié; ainsi, dans l'exemple suivant, la présence de stocks et de shares dans le contexte immédiat aidera à clarifier le sens de shorts, abréviation de short sellers, c'est-à-dire les vendeurs à découvert :

Some institutions make a point of buying heavily shorted stocks to force shorts to buy back the shares, driving prices up further. (Business Week, June 15, 1998, « How to Short a BullMarket -and Live »)

\subsection{L'approche phraséologique}

Si le travail d'initiation et de sensibilisation à la terminologie peut indéniablement aider à rapprocher davantage de l'authenticité en langue spécialisée et à mieux fixer les concepts, il est évident qu'il doit s'enrichir de l'apport que peut lui fournir l'observation des phrasèmes, c'est-à-dire ces combinaisons privilégiées par l'usage qui font que tel verbe, tel adjectif seront plus souvent mariés à une unité terminologique que d'autres. De la même manière que ces rapprochements consacrés par l'habitude existent dans la langue générale, ils sont présents dans la langue spécialisée. Cependant, les unions ainsi constituées ne sont pas systématiquement les mêmes et c'est pourquoi il importe de les observer et de les relever pour compléter les blocs déjà constitués par les unités terminologiques. Ce n'est pas parce que les adjectifs «mou» et "flasque» sont interchangeables dans certains contextes que l'on peut prendre sur soi de parler de "marché *flasque »; le même raisonnement vaut en anglais pour dull market qu'on ne peut prendre la liberté de transformer en *boring market. 
31 Au niveau des verbes qui accompagnent les termes, le principe est le même : la vigilance s'impose, mais une fois que le message est bien passé, travailler à ce niveau revient à créer des réflexes chez les apprenants, ce qui présente un double avantage : d'une part, ils découpent mieux les blocs de sens à la lecture, car ils s'attendent à trouver tel adjectif ou tel verbe en combinaison avec un terme clé qu'ils ont identifié, et d'autre part, à force de reconnaître, de retrouver ces tournures, ils vont se les approprier et les utiliser par euxmêmes dans leur production écrite et orale qui gagneront ainsi en qualité. Cette sensibilisation aux phrasèmes répond aux conseils des chercheurs qui se sont penchés sur les problèmes de lecture :

A final step at the simple recognition level is phrase identification - a first step toward the crucial skill of « chunking ", or reading in meaningful groups of words.

(Eskey \& Grabe 1992 : 233)

32 Nous ne voudrions pas donner la fausse idée que les phrasèmes sont limités aux groupes de l'unité terminologique à laquelle viennent s'ajouter un verbe ou un adjectif. En effet, il est des cas où le phrasème peut aller jusqu'à la phrase, notamment en ce qui concerne certains proverbes ou dictons, mais nous aurons l'occasion de revenir sur ce point ultérieurement. En revanche, nous aimerions souligner dès maintenant qu'à l'occasion d'un travail sur la phraséologie, il est déjà possible de relever certaines tendances à s'exprimer par images et qu'il est bon que les étudiants soient sensibilisés à ce phénomène. En effet, des études précédentes (Resche, 1997, 1998 a et b) ont démontré une nette tendance à la connotation métaphorique de certains phrasèmes pour parler de l'économie, des mouvements des marchés, des capitaux, des taux d'intérêt, de l'inflation et de la croissance, des monnaies pour ne citer que certains domaines.

En complétant les unités terminologiques, cette approche offre donc aux étudiants des segments de plus en plus longs sur lesquels ils peuvent se reposer dans leur lecture; insidieusement, s'ils se prennent au jeu de constituer des diagrammes de «champs phraséologiques ", ils seront amenés à lire davantage. Or, plus ils lisent, plus ils ont l'occasion de mettre en pratique les différentes techniques suggérées, et plus ils ont de chance de progresser dans leur approche des textes en langue étrangère spécialisée.

\section{Syntaxe}

34 Toutefois, l'étude des relations entre notions et termes, même complétée par l'observation des phrasèmes, ne suffit pas à permettre de maîtriser la langue si on laisse de côté la syntaxe. De toute évidence, il faut s'entendre sur cette approche : comme nous nous sommes appliquée à le rappeler au tout début de cette étude et dans le libellé du titre, il serait mal venu de parler de syntaxe de la langue spécialisée puisque son ancrage dans la langue générale est établi. Il convient plutôt de parler de l'usage que fait la langue spécialisée de la syntaxe, ce qui revient à observer et souligner les formes syntaxiques non pas caractéristiques de la langue spécialisée, mais privilégiées par cet aspect de la langue qui emprunte au fond commun. La tâche est assurément ambitieuse et ardue, car la langue spécialisée emprunte également à d'autres disciplines, comme c'est le cas pour l'économie qui a recours au discours mathématique dès qu'elle aborde l'analyse graphique ou la modélisation, par exemple. La question se pose parfois de délimiter clairement le terrain propre à telle ou telle spécialité...

Quoi qu'il en soit, l'approche syntaxique doit s'efforcer, en cernant avec plus de rigueur la langue spécialisée, de faciliter la reconnaissance par leurs pairs des futurs chercheurs que 
sont, par exemple, nos étudiants de troisième cycle en DEA. Ils seront en effet appelés à rédiger des articles en anglais et, de la qualité de leur expression, dépendra peut-être l'acceptation ou le rejet d'un article pour parution. Les traducteurs peuvent également bénéficier de cette approche qui leur permettra une plus grande authenticité de ton et de style.

Il va de soi que le cadre de cet exposé ne permet pas de développer comme il le faudrait cette voie d'accès à la langue spécialisée, dont l'analyse sera réservée à une autre étude, mais nous citerons en illustration quelques pistes à explorer, qui sont autant de points que les étudiants et les enseignants devront approfondir ensemble, dans la mesure où leur récurrence risque de constituer autant d'obstacles à la bonne compréhension de la chaîne écrite ou parlée et à une meilleure expression en langue spécialisée. Il importera par exemple de se pencher sur la question de la longueur des phrases, sur celle des groupes sujets et des noms et adjectifs composés, sur des procédés tels que la nominalisation, la passivation, sur des constructions verbales qui peuvent différer ponctuellement des constructions usuelles de la langue générale, ou encore sur l'utilisation inattendue de certains temps. Devront également faire l'objet d'une attention spéciale toutes les formes modales qui peuvent permettre de nuancer les affirmations, ce qui conduit à élargir le champ d'investigation pour prendre en compte les divers procédés de précautions oratoires.

Inévitablement, en abordant ces questions, on est amené à évoquer les notions de registres, de genres (Swales 1990), de types de discours. Il est utile de soumettre à une étude comparative aussi bien les articles de recherche que les articles de pseudo vulgarisation, mais également les ouvrages théoriques plus longs et les manuels destinés aux étudiants. Il faut également veiller à faire la part entre les formes syntaxiques fréquemment utilisées par un auteur précis et celles qui constituent un dénominateur commun aux spécialistes du domaine.

Pour éviter toute conclusion hâtive, des approches transversales s'avèrent indispensables : comparer plusieurs auteurs pour un même genre, plusieurs genres pour un même auteur, et peut-être aussi étudier l'évolution possible de la syntaxe chez un même auteur sur une période donnée. Sans prétendre établir un inventaire exhaustif des aspects syntaxiques sélectionnés par les spécialistes du domaine, une telle étude présente l'avantage de faire ressortir, le cas échéant, des constantes à travers les genres et les discours, et de souligner les divergences dues aux différences entre les publics cibles. Les conclusions devraient aider les futurs utilisateurs à mieux connaître et donc respecter les diverses contraintes de la langue spécialisée, contraintes de forme (abstracts, articles de recherche, etc.), contraintes de niveaux de langue et de niveau de technicité en fonction du lectorat ou de l'auditoire.

\section{Sensibilisation à la culture}

On peut entendre «culture » de deux manières différentes. Tout d'abord, puisque très souvent la langue est mise à contribution pour servir de support à une culture de groupe, " culture » peut faire allusion à la culture d'entreprise : une terminologie spéciale, dans ce cas, a pour rôle de souder le groupe qui l'utilise. Elle devient une sorte de sésame et il importe donc de la maîtriser pour pouvoir s'identifier à la communauté en question ou se faire reconnaître et accepter par elle. Vue sous un autre angle, cette même terminologie propre à un groupe peut donc avoir un aspect stratégique puisqu'elle ne sera pas 
systématiquement comprise par toute personne extérieure. Mais d'aucuns diront que l'approche terminologique et phraséologique est trop réductrice et assèche la langue spécialisée, en cherchant à créer des réflexes pavloviens chez les apprenants, ce qui risque de faire oublier tout ce qui fait qu'une langue vit et évolue, qu'elle peut s'exprimer de différentes manières. C'est la raison pour laquelle nous aimerions montrer comment l'approche de la termino-phraséologie peut déboucher sur autre chose, sur une autre acception du terme « culture » et ouvrir de nouveaux horizons.

\subsection{Proverbes et dictons}

Tout d'abord, comme nous l'avons laissé entendre précédemment, l'étude de la phraséologie ne se borne pas à constituer des listes de collocations de verbes et d'adjectifs accompagnant habituellement une unité terminologique ; il est en effet des phrasèmes qu'il est bon de relever en dépit du fait qu'ils ne portent pas spécifiquement sur un terme déjà répertorié, tout simplement parce qu'ils constituent des outils d'une autre sorte pour parler d'un domaine et comprendre les spécialistes qui les utilisent naturellement. Encore une fois, l'emprunt à la langue générale est à la source de ces phrasèmes que sont dictons et expressions à caractère proverbial qui permettent d'exprimer de manière courte et conventionnelle une opinion sur la situation, sur l'évolution probable de telle tendance, etc. Nous ne reviendrons pas sur les exemples déjà cités dans une autre étude et auxquels nous renvoyons le lecteur (Resche 1995 : 98-100), mais nous en citerons d'autres pour illustrer ce point : des conseils tels que buy the dips ou sell the rallies ne posent en effet aucun problème aux spécialistes et ne doivent plus en poser à nos étudiants qui les rencontrent dans les articles qu'ils étudient. L'intérêt que l'on peut trouver à ce travail est qu'il crée un lien entre les réalités techniques et une approche plus linguistique de la langue spécialisée. Les deux expressions ci-dessus sont automatiquement l'occasion de remarques sur le lexique et sur la syntaxe, les expansions évidentes étant : buy the stocks that have just plunged/ dipped/fallen et sell the stocks that have just recovered/gone back up/ rallied.

Considérons maintenant un autre type de commentaire : the trend is your friend. En dehors des discussions possibles sur le fond, avec des exemples et contre-exemples à l'appui, cette expression permet des remarques sur la rime interne et des comparaisons avec d'autres exemples plus généraux, mais pas toujours connus, du même phénomène ( $a$ friend in need is a friend indeed). Dans le même ordre d'idée, on pourra citer l'expression a spree a day keeps recession at bay rencontrée dans un article traitant du regain de confiance noté chez les consommateurs. Il va de soi que l'étudiant doit pouvoir apprécier le clin d'œil, et pour ce faire, connaitre le dicton de référence. Que dire encore de What comes up...? En l'occurrence, les points de suspension insistent sur la nécessité d'être capable de terminer seul par will go down pour signifier que « les arbres ne montent jamais jusqu'au ciel ». N'est-ce pas le même principe qu'une citation que l'on considère tellement connue que ce serait presque faire un affront à son public ou à son interlocuteur d'en donner la fin, du type : « ... ; mais aux âmes bien nées... »? (Corneille, Le Cid, II, 2). C'est comme s'il s'instaurait une certaine connivence entre initiés, d'où l'importance de maîtriser aussi cet aspect de la langue spécialisée. Indéniablement, un adage comme A rising tide lifts all boats, encore rencontré récemment à propos des hausses sur les différentes bourses mondiales, (The Economist June 6, 1998, p58) est une façon très ramassée (et métaphorique) de commenter le phénomène. Il est intéressant de noter que cette métaphore n'est pas 
seulement un effet de style caractéristique d'un journaliste, mais que le Professeur Paul Samuelson (1998: 37) y a recours tout naturellement dans son manuel Economics, lecture incontournable pour des étudiants en économie :

For most of American history, economic growth was a rising tide that lifted all boats, raising the incomes of the poor as well as those of the rich.

\subsection{La néologie} inévitablement les étudiants en face de termes nouveaux qui peuvent les dérouter par leur essence même et dont il importe donc de démonter les mécanismes avec eux afin de les aider à surmonter les difficultés de compréhension.

N'oublions pas que la néologie a plusieurs fonctions: d'une part, de manière générale, l'activité néologique répond à un besoin vital de dénommer de nouveaux concepts, de nouvelles techniques, des machines ou pièces détachées récemment apparues, et ceci est conforme à ce que nous avons dit de la fonction d'une terminologie donnée. D'autre part, et cet aspect est souvent lié au point que nous venons de mentionner, cette activité peut viser à normaliser des termes afin d'éviter un foisonnement d'appellations différentes. Ce dernier aspect n'exclut d'ailleurs pas une préoccupation pour la culture sous-jacente, puisque l'on conçoit qu'un terme mal perçu sera ignoré et que ce mauvais choix, s'il porte sur un objet commercialisé, risquera de faire perdre des ventes au profit de la concurrence. Mais là n'est pas notre propos.

Ce qui nous paraît particulièrement intéressant est l'émergence d'une néologie que nous qualifierons de "gratuite", puisqu'elle ne vise ni à combler un vide lexical, ni à normaliser l'usage de tel ou tel terme. Ce dernier aspect est particulièrement caractéristique de la néologie autour de l'euro, par exemple, dont l'étude fait ressortir qu'elle est non seulement le reflet des diverses opinions sur le sujet, mais aussi un formidable moyen d'influencer le public par le biais de connotations métaphoriques. La néologie devient alors le support d'un discours dont l'analyse aide à prendre le pouls d'une période donnée (Resche 1998a).

\subsection{Les métaphores}

La langue de l'économie n'est certes pas la seule à comporter des métaphores puisque, comme nous l'avons dit, la langue spécialisée plonge ses racines dans la langue générale qui est elle-même très métaphorique : nous parlons tous par métaphores, que nous en ayons conscience ou non. Cependant, on notera que le domaine économique est particulièrement riche sur ce plan, certainement parce que toute décision en matière de politique économique affecte directement ou indirectement le citoyen, qu'il s'agisse de l'inflation et des taux d'intérêt, du chômage, des impôts, du budget en général, de l'investissement, de la valeur de la monnaie, etc. Le discours métaphorique semble bien le plus sûr moyen d'expliquer des mécanismes parfois fort complexes, d'en minimiser l'importance si besoin est, de gagner la confiance en rassurant le public, d'influencer l'opinion, d'embellir la réalité aussi.

On pourrait bien sûr avancer que les métaphores sont l'apanage des outils de vulgarisation que sont les journaux et magazines, en notant toutefois au passage que, de la presse généraliste à la presse plus spécialisée, on relève les mêmes registres 
métaphoriques. Mais il faut se rendre à l'évidence, même les spécialistes et les techniciens recourent aux métaphores, et ils n'utilisent pas exclusivement ce type de discours pour se mettre à la portée des non-initiés, comme le montrent les exemples suivants. Le premier concerne l'impact sur l'économie américaine de la crise monétaire asiatique et son auteur en est le président de la Réserve Fédérale américaine, Alan Greenspan, qui parlait d'un avenir sombre: storm clouds massing over the western Pacific and heading our way (The Economist February 28, 1998). Les deux exemples suivants sont à replacer dans le cadre d'un séminaire du FMI, entre spécialistes pour lesquels tout effort de vulgarisation était superflu. Pourtant, pour décrire les changements survenus sur les marchés financiers du fait de la mondialisation, Lawrence Summers, éminent économiste de Harvard et soussecrétaire d'État au Trésor américain, a cru bon de choisir la métaphore des nouveaux supersoniques :

We can go where we want to go much more quickly, we can get there more comfortably, more cheaply and most of the time more safely, but the crashes, when they occur, are that much more spectacular. (The Economist, March 14, 1998, «OfTake-Offs and Tempests »)

47 À ce même séminaire, Joseph Stiglitz, économiste de renom à la Banque Mondiale, a, de son côté, dépeint les problèmes en termes nautiques :

Although an ill-repaired boat is more likely to sink, the force of powerful waves can cause even a perfectly sound vessel to founder. (The Economist March 14, 1998, « Of Take-Offs andTempests »)

Les hommes politiques ne sont d'ailleurs pas en reste, tel le premier ministre portugais qui, dans l'enthousiasme qui a suivi le sommet européen au cours duquel la future monnaie unique a été baptisée " euro ", s'est livré à une métaphore par laquelle il faisait de l'euro la première pierre d'un nouvel édifice (ou d'une nouvelle religion): the single currency will be the rock for Europe (The Economist December 23, 1995, "Single-Currency Minded»). Hans Tietmeyer a, lui aussi, eu recours au procédé métaphorique pour exprimer sa pensée au sujet de l'avenir de l'euro, en assimilant le lancement de la nouvelle monnaie au départ d'un train : it is better to delay departure than to derail outside the station. (Time April 1, 1996, "Caught in the Maastricht Grinder »). Enfin, ce sont une nouvelle fois les images nautiques qui ont été privilégiées par un administrateur de la Deutsche Bank pour donner son avis sur la question :

I'm afraid financial markets may soon begin to question whether it is all smooth sailing toward the euro or whether we're headed for the rocks. (Business Week February 17, 1997, « How Do You Short a Currency That Doesn't Exist ? »)

Passer sous silence ou escamoter cet aspect de la langue spécialisée reviendrait donc à en donner une vue incomplète et inexacte. Les étudiants doivent être en mesure de comprendre ces métaphores qui ont l'avantage de souligner d'une autre manière que le passage d'une langue dans une autre est aussi le passage d'une culture dans une autre. Comme l'a relevé Françoise Raby (1994 : 26),

La compréhension et l'appropriation de ces métaphores supposent de la part de l'étudiant la prise de conscience de modèles mentaux différents, à l'œuvre dans sa propre langue ou dans la langue cible. Il s'agit bien là d'un problème d'ordre culturel.

Par ce type d'approche, on arrive à conclure à l'existence de constantes dans les registres d'emprunt selon les domaines étudiés : c'est ainsi que bien souvent l'économie est décrite en terme de moteur qui chauffe trop ou au contraire s'enrhume, les capitaux et les monnaies en termes nautiques, etc. Certaines de ces métaphores passent inaperçues 
parce qu'elles sont lexicalisées ; certains les qualifient de mortes, mais nous préférons, pour notre part, les dire « en sommeil » car elles peuvent à tout moment être ravivées au détour d'une phrase, et surtout, elles servent de souches à des métaphores plus libres, mais appartenant au même domaine d'emprunt, et que nous avons baptisées « métaphore d'extension » (Resche, 1998b).

51 C'est en sensibilisant les étudiants à ce phénomène qu'on peut les aider à mieux le comprendre: s'ils sont déjà au fait des champs métaphoriques potentiellement exploitables, ils ne seront pas surpris par l'existence de ces variantes et les intégreront plus aisément au reste. Il importe, en effet, de souligner que ces métaphores sont structurées elles aussi en un double réseau: d'une part, on trouve le réseau métaphore souche/métaphores d'extension; d'autre part, on s'aperçoit que de nombreux registres sont interconnectés, ce qui se conçoit puisqu'il suffit d'un sème commun pour créer le lien. Les figures 4 et 5 suivantes permettront de visualiser ces réseaux :

Fig. 4. Réseau métaphore souche / métaphores d'extension

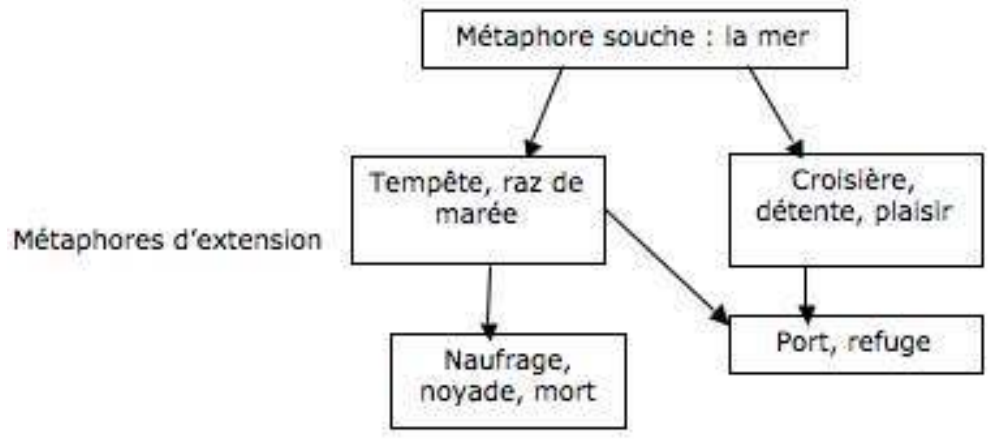

D'après la figure 4, la métaphore de la mer appliquée à une crise monétaire en Asie par exemple, et son impact sur les monnaies d'autres pays pourrait, selon qu'elles résistent plus ou moins bien, se développer en raz de marée saccageant tout sur son passage, donc en débâcle de grande envergure et il y aurait beaucoup de victimes, même si certains parvenaient à trouver refuge dans un port et évitaient ainsi de tout perdre; ou alors, cette crise pourrait n'affecter qu'un nombre limité de pays et les autres jouiraient d'une traversée très paisible et arriveraient sans problème au port. Il est d'ailleurs intéressant d'observer comment les métaphores peuvent s'entrelacer à partir d'un même point de départ, au point qu'il est possible de transcrire le lacis ainsi formé sous forme de schéma (Fig.5).

53 Les multiples liens qui unissent les métaphores entre elles passent, comme nous l'avons démontré pour l'euro (Resche 1998 b) par des sèmes communs: le sème de l'union se conçoit aisément pour le mariage ou le club, celui de la construction s'illustre à la fois dans le mariage, dans la création d'un club, et bien évidemment dans l'optique de la monnaie unique, dont la naissance sera le résultat d'un long cheminement, qui, au même titre que le mariage, peut être vu en termes religieux. Dans une autre perspective, la progression sur ce chemin passera par divers moyens de transport. Quant à la construction, elle emprunte naturellement au domaine du bâtiment, terme qui peut s'entendre soit comme un immeuble, soit comme un navire, auquel cas on rejoint la métaphore des transports. Dans tous les cas de figure, on remarquera que chaque métaphore peut s'envisager au positif comme au négatif: l'éventualité de l'échec est 
matérialisée dans le schéma par le dernier encadré ombré, dans lequel, selon les registres, elle s'exprime en termes de divorce, dissolution, effondrement, naufrage, déraillement ou accident.

Fig. 5. Lacis de métaphores à partir de la notion union monétaire

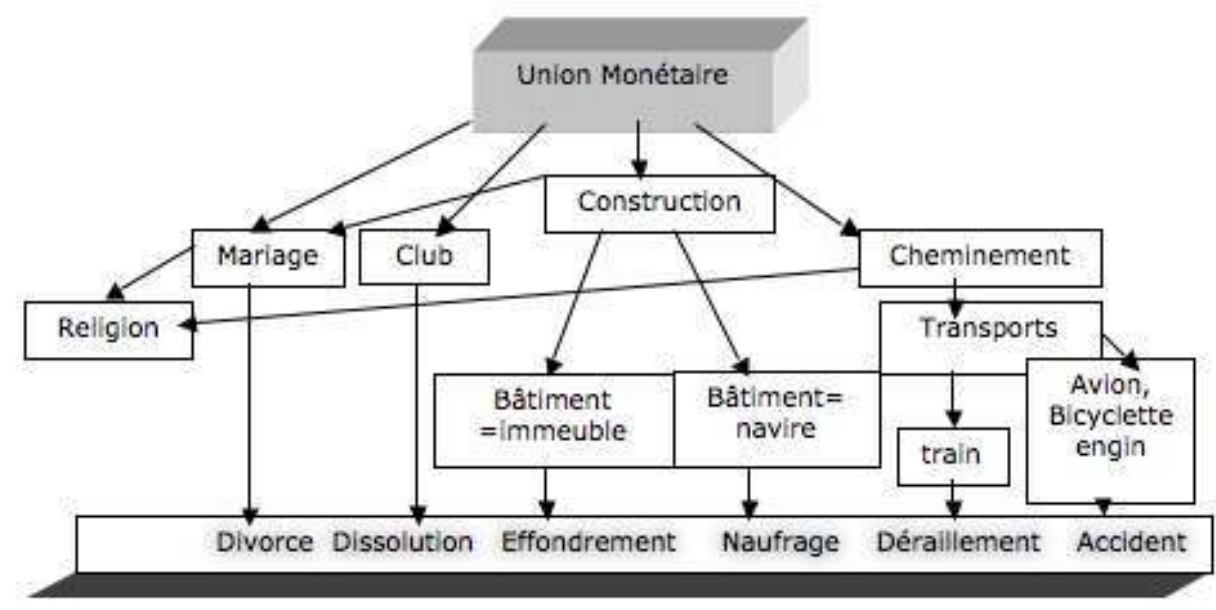

54 S'il est vrai que les métaphores permettent d'exprimer plus facilement des abstractions, elles ont également un autre effet, qui ressort lorsqu'on se livre à une analyse plus précise portant sur une période donnée: par le message qu'elles véhiculent, elles s'avèrent également être un miroir de l'évolution de la pensée et un moteur des opinions selon qu'elles se font l'écho des positions et le reflet des attitudes ou qu'elles influencent les réactions et pèsent sur les événements. C'est alors que le discours sous-jacent relèverait d'une analyse à caractère sociolinguistique. Cela entraîne bien au-delà de l'image de la langue spécialisée aride et réduite à un lexique étroit et rébarbatif! D'ailleurs, les clins d'œil à la littérature que l'on rencontre çà et là en travaillant les textes spécialisés prouvent que l'enseignement dans le secteur LANSAD permet d'autres ouvertures sur la culture.

\subsection{Les références d'ordre littéraire}

En dépit du sérieux des analyses économiques et des sujets abordés, il n'est pas rare de rencontrer des allusions à différents genres littéraires, qu'il s'agisse de contes pour enfants, de romans ou de pièces de théâtre. Les jeux de mots et pirouettes permis par ces renvois ne peuvent être compris que si les œuvres d'origine sont clairement identifiées par le lecteur.

Ainsi, un article intitulé Measure for Measure in Euroland risque de laisser perplexe l'étudiant qui n'aura pas reconnu la comédie de Shakespeare! Cet article porte, en fait, sur un risque de confusion pour le public si les gérants de fonds communs de placement s'avisent de profiter de l'occasion du passage à la monnaie unique pour retranscrire leurs comptes en euro en choisissant la méthode qui les avantagera :

A report published by the Bank of England on June $11^{\text {th }}$ warns that unit-trust (mutual-fund) managers might seize the chance to rewrite some history. They have ample room to beautify their records, by using different ways to translate past returns, earned in national currencies, into euros. (The Economist, June $13^{\text {th }}, 1998$ ) 
57 Inflation Trouble, sinon qu'il fournit une occasion inespérée de familiariser des étudiants en anglais économique avec les sorcières de Macbeth? (IV, 1, 10-11: Double, double toil and trouble : Fire burn; and, cauldron, bubble)

Ce sont bien souvent ces allusions, plus que le contenu de l'analyse économique, qui déroutent les étudiants parce qu'ils ne possèdent pas les références nécessaires pour apprécier ces procédés. Il importe donc d'expliciter les allusions, de combler les lacunes éventuelles en soulignant les liens étroits que la langue entretient avec la civilisation et la culture, de faire apprécier le cas échéant, les allitérations, les jeux de mots, les néologismes, les métaphores qui sont ainsi filées.

Un article portant sur les fluctuations boursières liées aux remous engendrés pas la crise asiatique (The Economist 24 Octobre 1998), et qui titrait «Once Upon a Time in Wall Street ", illustre bien les difficultés que peut représenter un constant maillage entre réalité et fiction, même si, d'entrée, l'allusion au conte de fée est évidente :

Stockmarket investors are fond of fairy tales. Until this summer, their favourite was Goldilocks: the American economy, they claimed, was neither too hot, nor too cold, but just right. Pity they forgot that, in the story, Goldilocks was eventually chased away by the bears. After the markets fell back in July, the favourite tales changed: the recent talk has been of something nasty in the woodshed. But as in all good stories, a fairy godmother has arrived in the nick of time. With a flick of his magic wand, and two swift cuts in interest rates, Alan Greenspan has saved the day...

Le paragraphe suivant, intitulé Alan in bubbleland poursuit dans cette même voie, et l'on peut notamment y lire que, contrairement aux contes de fées, les bulles financières n'ont jamais de fins heureuses... Il faut préciser que, depuis, l'expression the Goldilocks economy ,qui, dans un premier temps, pouvait certes être considérée comme un effet de style recherché par le journaliste, a été banalisée et quasiment lexicalisée.

61 D'ailleurs, la presse n'a pas le privilège de ces allusions: d'autres genres, comme par exemple l'ouvrage de recherche, empruntent également à la littérature. Ainsi, Gary Becker, professeur d'économie à l'université de Chicago et ancien conseiller économique auprès du président Clinton, n'a pas hésité à emprunter à Jane Austen (en s'autorisant une variante) et à Dostoievski pour donner un titre à deux de ses chapitres Price and Prejudice et Crime and Punishment dans un très sérieux ouvrage $(1976$ : 17, 39). Robert J. Barro, professeur d'économie à Harvard et Vittorio Grilli, professeur spécialiste de la finance à l'université de Londres (1994: 16) insistent, pour leur part, sur le parallèle devenu classique avec le héros de Daniel Defoe pour mieux faire passer leur message et n'hésitent pas à choisir pour titre à leur chapitre 2 : The Economics of Robinson Crusoe :

We examine the choice problems of an isolated individual, similar to the Robinson Crusoe of Daniel Defoe's novel. We assume that Crusoe's choices are guided by enlightened self-interest, that is, we exploit the central economic postulate of optimizing behaviour... By studying Crusoe's behaviour, we can understand the trade-off between leisure and consumption that applies in complicated market economies...When goods cannot be stored over time, Crusoe's budget constraint dictates that his consumption equals his production.

Paul Samuelson (1998: 712), quant à lui, n'hésite pas à citer le poète Robert Frost pour faire réfléchir les étudiants d'économie sur les conséquences du protectionnisme en matière de commerce international :

Before I built a wall I'd ask to know

What I was walling in or walling out... 
Ce poète lui permet également d'illustrer la question du coût d'opportunité (1998 : 129)

Opportunity cost, then, is a measure of what has been given up when we make a decision. Consider what Robert Frost had in mind when he wrote :

Two roads diverged in a wood, and I -

I took the one less traveled by,

And that has made all the difference.

64 Est-ce à une vie citadine que Frost pensait en faisant allusion à cette autre voie ? Samuelson demande alors au lecteur de réfléchir au coût d'opportunité qu'un tel autre choix aurait entraîné. Si Frost avait emprunté un chemin bien frayé, s'il n'avait pu être inspiré par la nature, parler des murs, des bois, des arbres, nous aurions, certes, tous beaucoup perdu.

Dans ce même manuel d'économie générale, il emprunte également à Hemingway dans un paragraphe intitulé For Whom the Bell Tolls (p. 4) puis à nouveau en écho dans son chapitre sur la globalisation des échanges (p. 712)

Economically, no nation is an island unto itself. When the bell tolls depressions or financial crisis, the sounds reverberate around the world.

Robert Burns y est également à l'honneur avec un écho, conscient ou non, au poème To $a$ mouse (The best-laid schemes o' mice an' men / Gang aft agley, / An' lea'e us nought but grief an'pain / For promis'd joy!):

Others have fallen behind, either because they are trapped in the vicious cycle of poverty or because war, corruption, and the heavy hand of government regulation have snuffed out the flames of entrepreneurship and wrecked the best-laid plans.

On le voit, tant au plan des dictons et proverbes que des néologismes, des métaphores ou des références littéraires, les langues spécialisées " peuvent faire preuve de vitalité et de créativité lorsqu'elles dépassent le jargon réducteur du spécialiste pour puiser aux sources de notre civilisation » (Lavault $1994: 53$ )

\section{Conclusion}

Il ressort de cette étude que, s'il n'existe pas de formule magique pour mieux appréhender la langue spécialisée, il se trouve néanmoins plusieurs voies d'accès que nous avons essayé d'envisager et qui peuvent être empruntées tour à tour ou conjointement en fonction des besoins et du niveau de chacun. L'utilité des cours de langues (et surtout de l'anglais) dans le secteur qui est le nôtre n'est plus à démontrer à l'heure de la mondialisation des échanges, non seulement sur le plan commercial, mais également en matière de recherche scientifique, au sens large du terme. Qu'on le regrette ou qu'on s'en réjouisse, pour se tenir au courant de l'évolution des idées, des techniques et des disciplines, et pour trouver leur place dans la communauté de spécialistes qu'ils sont appelés à rejoindre, les futurs chercheurs, scientifiques, économistes, juristes, etc. devront maîtriser l'anglais général et l'anglais spécialisé.

Aborder les langues spécialisées dans toute leur complexité devient donc une priorité pour un large éventail de publics dont le nombre va croissant. Nous avons d'abord voulu montrer comment la prise de conscience des différents processus exploitables pouvait faciliter la lecture de textes spécialisés, en considérant non seulement l'objectif immédiat de l'enseignement dans le cadre universitaire, mais également une perspective plus lointaine dans laquelle nos étudiants devront être autonomes dans leur découverte de la langue spécialisée écrite, soit dans la suite de leurs études, soit dans le cadre de leur futur 
emploi. Nous avons ensuite essayé de faire ressortir les diverses facettes de la langue spécialisée, non seulement techniques mais également culturelles, en explorant les multiples réseaux dont elles sont formées: réseaux de termes et de collocations, de phrases et de paragraphes, réseaux de néologismes, dictons et proverbes, et, pour terminer, réseaux de métaphores, de références culturelles, y compris littéraires. Si nous sommes passée plus rapidement sur les réseaux syntaxiques, c'est parce qu'ils demandent à être explorés plus étroitement, ainsi que les différents genres dont les contraintes doivent être prises en compte par les divers utilisateurs de la langue spécialisée. Ceci doit constituer un futur axe de travail pour que l'étude de la langue spécialisée soit plus complète et plus précise. Nous espérons simplement avoir ici contribué à donner de la langue spécialisée l'image d'une langue qui vit et avoir restitué la richesse de ses trames.

\section{BIBLIOGRAPHIE}

Barro, J. Robert \& Vittorio Grilli. 1994. European Macroeconomics. Londres : The MacMillan Press Ltd.

Becker, Gary. 1976. The Economic Approach to Human Behaviour. Chicago, IL : The University of Chicago Press.

Carrell, Patricia L. 1992. « Interactive text processing: Implications for ESL/second language reading classrooms ». In Carrell, Patricia, Joanne Devine \& David Eskey (dir.). Interactive Approaches to Second Language Reading, 239-259.

Eskey, David \& William Grabe. 1992. «Interactive models for second language reading perspectives on instruction ». In Carrell, Patricia, Joanne Devine \& David Eskey (dir.). Interactive Approaches to Second Language Reading, 223-238,

Fade, Pascale. 1994. « Macro-structure et spécialité dans les textes de sciences humaines ». ASp 5-6, 121-127.

Guilbert, Louis. 1973. «La spécificité du terme scientifique et technique ». Langue Française 17, Les vocabulaires techniques et scientifiques, 5-17.

Lakoff, George \& Mark Johnson. 1980. Metaphors We Live By. Chicago, IL : University of Chicago.

Lavault, Elisabeth. 1994. « Du téléphone à micro-ondes au cheval de Troie : quelle culture pour le traducteur spécialisé ? ». ASp 5-6, 45-54.

Lerat, Pierre. 1995. Les langues spécialisées, Paris : Presses Universitaires de France.

Lerat, Pierre. 1997. « Approches linguistiques des LSP ». ASp 15-18, 1-10.

Perrin, Michel. 1990. « De l'utilisation 'communicative' des documents authentiques ». Actes du $\mathrm{XI}^{\mathrm{e}}$ Colloque du GERAS, Bordeaux, 11-33.

Raby, Françoise. 1994. « Les relations entre savoirs linguistiques et savoirs de spécialité dans la construction des processus de passage de l'être 'cultivé' à l'être civilisé ». ASp 5-6, 21-30.

Resche, Catherine. 1995. « La langue de la Bourse : convergences et divergences ». ASp 7-10, 91-112. 
Resche, Catherine. 1996. «La terminologie comparée au service de l'enseignement en langue de spécialité en 3è cycle : DEA Monnaie-Finance-Banque ». ASp 11-14, 195-206.

Resche, Catherine. 1997. «Prolégomènes à la phraséologie comparée en langue de spécialité : exemple de l'anglais et du français de la finance ». ASp 15-18, 487-504.

Resche, Catherine. 1998a. « De l'Europe à l'euro, ou 'l'industrieuse activité' de la langue : recensement et analyse des néologismes à partir de Euro/euro de 1991 à 1998 ». ASp 19-22, 185-208.

Resche, Catherine. 1998b. « Discours métaphorique et monnaies : les particularités de l'euro ». ASp 19-22, 67-88 .

Samuelson, Paul A. \& William D. Nordhaus. 1998. Economics. Irwin McGraw-Hill, Sixteenth Edition. Stanovitch, K.E. 1980. « Toward an interactive-compensatory model of individual differences in the development of reading fluency ». Reading Research Quarterly 32-71.

Swales, John, M. 1990. Genre Analysis. Cambridge : Cambridge University Press.

\section{RÉSUMÉS}

Enseigner l'anglais dans le secteur LANSAD ne doit pas faire perdre de vue la langue générale dans laquelle la langue spécialisée est enracinée, même s'il est évident qu'il faut s'attacher à donner aux étudiants la possibilité d'aborder un secteur spécialisé et d'en maîtriser les moyens d'expression. La présente étude passe précisément en revue plusieurs techniques qui sont autant de voies d'accès à la langue spécialisée : d'abord, la lecture, voie privilégiée pour aborder les textes spécialisés dans de bonnes conditions et améliorer leur compréhension, puis l'approche terminologique, et son complément naturel, la phraséologie. Cette étude des notions et des collocations qui tissent une toile autour des termes noyaux doit être complétée par un examen de la syntaxe, qui permet de souligner les formes privilégiées par la langue spécialisée. Enfin, la dimension culturelle aide, au travers de l'initiation à l'observation des néologismes, des dictons et proverbes, des nombreux réseaux de métaphores, sans oublier les références à la littérature, à souligner que l'étude de la langue spécialisée n'est pas aussi réductrice et rébarbative que certains voudraient le faire croire.

It is now widely acknowledged that ESP should not be considered as a sub-language for it is deeply-rooted in general-purpose English indeed. Teaching ESP therefore implies a wide range of access routes, which the present paper offers to explore. Its first focus, reading, is examined from different angles; bottom-up, top-down and interactive approaches are thus reviewed. Then, terminology and phraseology are dealt with, the former in terms of an organized network of notions and the latter as a set of potential collocates that build a web around the core units. However, the analysis would not be complete if the syntactic forms privileged by ESP were not investigated. Finally, through the study of neologisms, proverbs, metaphors and various references to literature, the cultural dimension is emphasized with a view to proving that considering ESP as a mere series of technical words to be mastered would be giving an oversimplified, biased and unfair view of what it really is. 
INDEX

Mots-clés : culture, langue spécialisée, lecture, métaphore, néologie, phraséologie, terminologie

Keywords : ESP, metaphor, neology, phraseology, reading, terminology

\section{AUTEUR}

\section{CATHERINE RESCHE}

Catherine Resche est maître de conférences à l'université de Paris 2 Panthéon-Assas, où elle est plus particulièrement chargée de l'enseignement de l'anglais en $3^{\mathrm{e}}$ cycle : DEA Monnaie-BanqueFinance, DESS Techniques Bancaires et Financières, Magistère Banque-Finance, DESS Commerce International. Elle est membre du Cerlac (Centre de Recherche en Langues et Cultures) de l'Université de Paris 1- Panthéon-Sorbonne. catherineresche@club-internet.fr 